\title{
Common genetic variation within miR-146a predicts disease onset $\&$ relapse in multiple sclerosis
}

Yuan Zhou ${ }^{1^{\star}}$, MS; Ming Chen ${ }^{2 *}$, MS; Steve Simpson, Jr. ${ }^{1}$, PhD; Robyn M Lucas ${ }^{3}$, PhD; Jac C Charlesworth ${ }^{1}, \mathrm{PhD}$; Nicholas Blackburn ${ }^{1}, \mathrm{PhD}$; Ingrid van der $\mathrm{Mei}^{1}$, PhD; Anne-Louise Ponsonby ${ }^{4}$, PhD; Ausimmune/AUSLONG investigators group ${ }^{5}$; Bruce V Taylor $^{1}, \mathrm{MD}, \mathrm{PhD}$

${ }^{1}$ Menzies Institute for Medical Research, University of Tasmania, Hobart, Australia; 2Department of Clinical Laboratory, Xinqiao Hospital, Third Military Medical University, Chongqing, China; ${ }^{3}$ National Centre for Epidemiology and Population Health, Research School of Population Health, The Australian National University, Canberra, Australia; ${ }^{4}$ Murdoch Childrens Research Institute, University of Melbourne, Melbourne, Australia; ${ }^{5} \mathrm{~A}$ full list of members is provided in the Acknowledgments.

* These authors contributed equally.

Corresponding author: Bruce V. Taylor

Corresponding author's address: 17 Liverpool Street, Hobart, TAS 7000, Australia Corresponding author's phone and fax: 0362267700 Corresponding author's e-mail address: Bruce.Taylor@utas.edu.au 


\begin{abstract}
Background: Despite extensive studies focusing on the changes in expression of microRNAs (miRNAs) in multiple sclerosis (MS) compared to healthy controls, few studies have evaluated the association of genetic variants of miRNAs with MS clinical course.
\end{abstract}

Methods: We investigated whether a functional polymorphism in the MS associated miR-146a gene predicted clinical course (hazard of conversion to clinically definite MS (CDMS) and/or relapse, and annualized change in disability), using a longitudinal cohort study of persons with a first demyelinating event followed up to their 5-year review.

Results: We found the genotype $(G C+C C)$ of rs2910164 predicted relapse compared with the GG genotype (HR=2.09 (95\% Cl: 1.42, 3.06), $p=0.0001)$, as well as a near-significant $(p=0.07)$ association with CDMS conversion risk. Moreover, we found a significant additive interaction between rs2910164 and baseline anti-EBNA-1 IgG titers predicting risk of conversion to CDMS (relative excess risk due to interaction [RERI]: 2.39, $p=0.00002)$ and of relapse (RERI: $1.20, p=0.006)$. Supporting these results, similar results were seen for the other EBV-correlated variables: anti-EBNA-2 IgG titers and past history of infectious mononucleosis. There was no association of rs2910164 genotype for disability progression.

Conclusion: Our findings suggest miR-146a and its target genes are potential therapeutic targets for modulating clinical course in MS. 
Keywords: miR-146a, clinical definite MS, relapse, EDSS 


\subsection{Introduction}

MicroRNAs (miRNAs) are a family of 21 to 25 nucleotide-long noncoding small RNAs that regulate gene expression[1]. Despite extensive studies focusing on changes in the expression of miRNAs in multiple sclerosis (MS) compared to healthy controls[2], few studies have sought to determine the function of genetic variation in miRNAs genes, or to assess whether such variation could predict MS clinical course, directly or by interaction with other risk factors.

One particular miRNA (miR-146a) has been investigated for its role in MS. miR-146a is primarily involved in the regulation of inflammation[3] and it is up-regulated in active MS brain lesions[4]. In MS patients with relapsing remitting disease, miR-146a expression was significantly lower in those treated with glatiramer acetate than in treatment-naïve patients[5]. Additionally the expression of miR-146a is altered by infection with Epstein-Barr virus (EBV)[6] and smoking[7], both of which are wellknown MS risk factors[8, 9]. For these reasons, miR-146a is a biologically plausible candidate genetic risk locus in MS.

The minor allele C of rs2910164, which is the only common SNP located within the miR-146a gene (chr5: 159,912,359-159,912,457), causes mis-pairing within the hairpin of pre-miR-146a (Figure 1) and may decrease the expression of mature miR$146 a[10]$ either by reducing the stability of the pri-miRNA, the efficiency of processing of pri-miRNA into pre-miRNA, or the efficiency of processing the premiRNA into the mature miRNA. Although rs2910164 is the only common variant within the miR-146a gene, there are other rare variants that may significantly 
influence miR-146a expression. However, we are unable to assess the effects of these rare variants due to our sample size. Given the potential for both direct and interactive relationships of miR-146a with MS risk and clinical course, as well as the impact of this polymorphism on the expression and function of miR-146a, we developed an a priori hypothesis that this polymorphism may be an important modulator of MS clinical activity.

We therefore undertook a study of clinical outcomes in a well-established, prospectively followed cohort of patients who were first recruited after diagnosis of a first demyelinating event (FDE) or initial diagnosis of progressive disease, to assess the effect of this functional genetic variation in the miR-146a gene on the transition to clinically definite MS (CDMS), relapse frequency and disability progression.

\subsection{Methods}

\subsubsection{Study design}

The Ausimmune Longitudinal (AusLong) Study builds upon the original Ausimmune case-control study[11] that sought to elucidate environmental, genetic and personal risk factors for the onset and progression of MS. The Ausimmune Study recruited 282 participants with a first clinical diagnosis of central nervous system demyelination, including 216 participants with a classic FDE (negative history for an undiagnosed event prior to commencement of recruitment) indicating high risk of developing MS[12]. At 5-year follow-up, the diagnosis for three participants had been changed to a non-MS illness; 258 of the original case participants were determined 
to have had bout-onset disease (169 of these participants had an FDE just prior to study recruitment, and 69 had an FDE in the more distant past). The remaining 20 participants were now determined to have presented with primary progressive MS (PPMS). Since 2008, the AusLong Study has followed case participants of the Ausimmune Study (retention rate 84.6\%); many participants have now been followed for over ten years since the event that brought them into the study. The present analysis is for the period from FDE/recruitment to the 5-year review, as this is the most recent face-to-face review which all currently enrolled participants have completed, thus allowing greater uniformity in clinical follow-up.

The Ausimmune Study and AusLong Study were approved by nine regional Human Research Ethics Committees. All participants gave written informed consent.

\subsubsection{Exposure \& clinical course measures}

The Ausimmune Study and subsequent reviews as part of the AusLong Study collected data on environmental and behavioural parameters including history of infectious mononucleosis (baseline only) and smoking. Anti-EBNA-1 IgG and antiEBNA-2 IgG were measured in serum samples collected at baseline, using immunofluorescence assays: anti-EBNA-1 using a commercial ELISA (DiaSorin) and anti-EBNA-2 using an in-house ELISA whose protocol is described elsewhere[13]. Several clinical outcomes were evaluated, including conversion to CDMS, occurrence of relapse, and annualized disability progression from FDE to five-year review. 
Conversion to CDMS was defined primarily as the occurrence of two or more clinical demyelinating episodes, thus satisfying the diagnostic requirements of dissemination in space and time, or a single episode plus paraclinical evidence, as per the 2005 McDonald criteria[14] (a minority of cases were diagnosed following MRI based on this latter criterion $(n=20))$. Conversion to CDMS was reported at annual review and cross-checked with neurological records. A relapse was defined according to the 2001 McDonald Criteria[15] as the acute or sub-acute appearance or reappearance of a neurological abnormality (lasting at least 24 hours) in the absence of other potential explanatory factors. Relapses were reported at annual review or derived from medical records, and only relapses which were diagnosed and verified by a neurologist were included in this analysis. Disability was assessed by the Kurtzke Expanded Disability Status Scale (EDSS)[16], assessed at the 5-year review; the EDSS on the day before FDE was assumed to be 0 . For the participants with a diagnosis of primary progressive presentation, where the date of disease onset was more difficult to define and disability levels are not associated with relapse, the annual change in disability was taken as the difference between the baseline recorded EDSS and the 5 year EDSS.

\subsubsection{Genotyping}

Genotyping for rs2910164 was performed using the predesigned $\operatorname{TaqMan}^{\circledR}$ genotyping assay C_15946974_10 from ThermoFisher Scientific (catalog number 4351379). Samples were genotyped in 96-well plates on a Roche LightCycler ${ }^{\circledR} 480$ Real-Time PCR system. Per sample $7 \mu \mathrm{L}$ reaction volumes were used with $1 \mu \mathrm{L}$ of DNA at $10 \mathrm{ng} / \mu \mathrm{L}, 4 \mu \mathrm{L}$ of TaqMan ${ }^{\circledR}$ Genotyping Master Mix (Applied Biosystems ${ }^{\mathrm{TM}}$ ), 
$2.9 \mu \mathrm{L}$ of nuclease free $\mathrm{H}_{2} \mathrm{O}$ and $0.1 \mu \mathrm{L}$ of $40 \times$ TaqMan genotyping probe. $5.9 \%$ of samples were genotyped in replicate with $100 \%$ replication.

\subsubsection{Data analysis}

Predictors of time to conversion to CDMS and of relapse were evaluated by Cox proportional hazards regression models, the latter for repeated events[17]. All covariates satisfied the proportional hazards assumption, excepting study site in the relapse analysis. Accordingly, all relapse analyses were stratified on study site.

Predictors of annualized change in EDSS were evaluated using linear regression, adjusted for whether persons were having a relapse at the time of their 5-year EDSS assessment. Because the annualized change in EDSS was highly skewed, a logtransformation was applied to satisfy linear regression assumptions of minimal heteroskedasticity. All means and coefficients, however, were back-transformed and presented on the original scale of the change in EDSS variable.

Interaction was assessed in two fashions, multiplicative and additive. Multiplicative interaction was assessed by generating a product term of the two variables to be assessed, with the significance of this two-component term delineating the interpretation of the significance of the interaction. Additive interaction was assessed by generating a four-level categorical term equal to 0 where both genetic and environmental risk factors were not present (the background risk $\left(r_{B}\right)$ ), equal to 1 and 2 where only the genetic or environmental risk factors were present ( $r_{G}$ and $\left.r_{E}\right)$, and equal to 3 where both genetic and environmental risk factors were present (rGE). 
When evaluating a continuous environmental factor, a dichotomous variable was generated by dividing the sample at the median of the continuous term. The relative excess risk due to interaction (RERI) on the additive scale was calculated by subtracting the sum of risk measures (e.g. hazard ratios, HRs) for the individual exposures $\left(r_{E}+r_{G}\right)$ from the risk measure for the combined exposure ( $\left.r_{G E}\right)$ and adding the background risk $\left(r_{B}=1.00\right)$ (i.e., $\left.R E R I=H R_{G E}-\left(H R G_{G}+H R E_{E}\right)+1\right)$. The statistical significance of this RERI (probability of a risk of this magnitude occurring due to chance alone) was evaluated by a permutation simulation, randomly redistributing participants to one of the four levels of the additive interaction term in proportion to their original distribution. For example, the proportions of the four-level EBNA1-miRNA interaction term $(32.5 \%, 31.5 \%, 19.8 \%, 16.2 \%)$ were retained in the simulation, but redistributed randomly amongst the participants who had data on both EBNA1 and miRNA genotype. These simulated interaction terms were generated, analysed and the magnitudes of the estimates resulting were retained. These simulations were run 50,000 times and the proportion of magnitudes resulting that were as or more extreme than that found in the as-measured analyses denoted the significance of the interaction.

All statistical analyses were conducted in Stata/SE 12.1 (StataCorp LP, College Station, Texas, USA).

\section{$1.3 \quad$ Results}

Of the 279 case participants in the Ausimmune/AusLong Study, 151 participants had a classic FDE that is those who had their diagnostic events during the follow-up period (given the importance of accurate dates in survival analysis, restriction to 
these individuals is appropriate) and who had genotype data, the distribution of female sex ( $n=117,77.5 \%)$, age at baseline (mean: 37.4, SD: 9.5), conversion to CDMS $(n=83,55.0 \%)$, relapse number $(n=198)$ and 5-year EDSS (median: 1.25, IQR: 0 -2) were similar to the parent group, excepting relapse number which was a function of the lower clinical activity in this group, some of whom were not yet CDMS at the 5 year review.

\subsubsection{Risk genotype $(G C+C C)$ of rs2910164 directly predicts progression to CDMS and relapse}

Among the group of participants whose FDE occurred immediately prior to recruitment to the Ausimmune Study $(n=151)$ and who were thus at risk of a second event diagnostic of CDMS within the period after referral to the study, we found that having genotype $(\mathrm{GC}+\mathrm{CC})(\mathrm{N}=93)$ of rs2910164 compared to $\mathrm{GG}(\mathrm{N}=58)$ was associated with an increased hazard of converting to CDMS (HR: 1.52 (95\% Cl: $0.97,2.42), p=0.07$, Table 1). A stronger result was seen for relapse (HR=2.09 (95\% $\mathrm{Cl}: 1.42,3.06), p=0.0001)$, partly reflecting the greater number of events $(n=83$ CDMS vs. 198 relapses). Figures $2 a$ and $2 b$ show the survival curves for the two genotypes for CDMS and relapse, respectively. The rs2910164 genotype did not predict annualized change in EDSS ( $p=0.25$ Table 1$)$. In the subgroup analysis only including female $(\mathrm{N}=117)$, more significant associations were observed for conversion to CDMS (HR=1.68, $\mathrm{p}=0.04)$ and of relapse $(H R=2.30, p=0.00006)$. 


\subsubsection{Interactions for rs2910164 with EBV related variables in predicting CDMS and relapse}

The rs2910164 SNP did not significantly interact with EBV-related parameters (serological, clinical) on the multiplicative scale in predicting either CDMS or relapse (Table 2). We did, however, find a significant interaction on the additive scale between rs2910164 and baseline anti-EBNA-1 IgG titers predicting risk of conversion to CDMS (RERI: 2.39, p=0.00002) and relapse (RERI: 1.20, p=0.006) (Figure 3, Table 2). The combined effect of having both a high baseline anti-EBNA-1-IgG titer and the risk genotype $(\mathrm{GC}+\mathrm{CC})$ of $\operatorname{miR}-146 a(\mathrm{~N}=24)$ was higher than expected based on the effects of having high baseline anti-EBNA-1-IgG titer in the absence of risk genotype $(\mathrm{GC}+\mathrm{CC})$ of miR-146a $(\mathrm{N}=42)$, and the effects of having risk genotype $(\mathrm{GC}+\mathrm{CC})$ of $m i R-146 \mathrm{a}$ in the absence of high baseline anti-EBNA-1-IgG titer $(\mathrm{N}=28)$. Supporting these results, similar results were seen for the other EBV correlated variables: anti-EBNA-2 IgG titers and having a history of infectious mononucleosis (Table 2).

No significant interactions were observed with smoking (data not shown).

\subsection{Discussion}

Here we have shown that the risk genotype $(\mathrm{GC}+\mathrm{CC})$ of miRNA-146a SNP rs2910164 directly predicts conversion to MS and risk of relapse but not 
accumulation of disability. We also provide evidence of an interaction on the additive scale, between the risk genotype of rs2910164 and baseline-measured EBV-related serological and clinical variables in predicting CDMS and relapse, such that these environmental factors are significantly more strongly associated with clinical outcomes among those carrying the miR-146a risk variant.

The role of miRNAs in the development and intensity of inflammatory reactions in complex disorders such as MS is now being explored. Increased expression of miR146a has been found to be associated with a more intense inflammatory state in MS brain lesions[4] and to be down-regulated by treatment with glatiramer acetate[5]. Jazdzewski et al found that the C allele of rs2910164 demonstrated lower expression compared with the wild-type G allele using transfected cells[18]. In MS, patients with the C allele of rs2910164 (compared to those with the GG and GC alleles) had higher expression of the prototypical pro-inflammatory cytokines TNF- $\alpha$ and IFN- $\gamma$ in peripheral blood mononuclear cells[19]. Such changes were modulated by miR-146a through signaling pathways targeting TRAF6 and IRAK1 gene expression, which establishes a negative feedback loop that controls the intensity and the duration of NF-kB signaling.

In the work by Li et al[19], they demonstrated that rs2910164 is associated with MS risk in female patients only compared with healthy controls. In our subgroup analysis only including females, we further substantiated this finding, in that the association of conversion to CDMS and hazards of relapse were more pronounced in females compared to males. As MS is up to 3 times as common in females, this disparity may be explained by sex hormones, especially estrogen, which has been shown to 
increase secretion of proinflammatory cytokines[20]. Additionally, in estrogen treated splenic lymphocytes, miR-146a expression was decreased compared to placebo controls[21].

The role of this miR-146a polymorphism in predicting other autoimmune diseases has also been investigated, however, the results were inconclusive[22]. In a recent meta-analysis, researchers found that rs2910164 was associated with a decreased risk for developing psoriasis and asthma, but had no association with rheumatoid arthritis and systemic lupus erythematosus risk[22]. Such differences are also reflected in miR-146a expression studies. For example, similar upregulation of miR146a expression has been found for other autoimmune diseases such as rheumatoid arthritis and psoriasis, but in systemic lupus erythematosus, miR-146a expression was downregulated[23]. These difference may be due to the fact that the expression of miRNA changes may depends on sampling time (samples collected from acute stage vs. inactive stages of disease) and tissue type analyzed, or that changes in miR-146a expression are disease specific.

Previous work has shown links between miR-146a and EBV infection. Using EBV de novo infection of primary cultured human B cells, miR-146a was down-regulated on initial infection, but was dramatically up-regulated upon induction of the lytic cycle[6]. In T-cells, decreased expression of miR-146 can cause hyper-responsiveness to Tcell receptor signals and unresolved T-cell-mediated inflammation[24]. Several studies have demonstrated the importance of T-cell driven immune responses in the association between EBV infection and onset of MS[25]. These effects of EBV infection on miR-146a expression, and on the effectiveness of T-cell responses, 
provide plausibility to enhanced effects on disease course of combined variation in miR-146a genotype and markers of EBV infection.

In our analyses we demonstrated an additive interaction between EBV infection and miR-146a genotype. Similar additive interactions have been previously demonstrated between markers of EBV infection and the HLA-DRB1*15 genotype[26, 27] and have been interpreted as indicating that the two factors are component causes of the same sufficient cause for a substantial proportion of people who are in the at-risk category of each factor - in this case the $(\mathrm{GC}+\mathrm{CC})$ genotype and a higher than median anti-EBNA-1 IgG level. We did not find a significant multiplicative interaction. We also did not observe interaction between smoking and miR-146a genotype. However, interaction between smoking and genetic factors in predicting MS risk has been controversial. In both of the above studies[26, 27] there was no significant additive interaction between smoking and $H L A-D R B 1 * 15$ genotype in predicting MS risk. On the contrary, work by Anna et al.[28] observed an additive interaction between passive smoking and carriage of $H L A-D R B 1 * 15$ risk genotype.

The strengths of our study include that we have detailed information on the clinical course from the first diagnosis of CNS demyelination for over five years, with high cohort retention. Only medically confirmed relapses were included in the analysis. One limitation is that the sample size of participants with a FDE close to the time of recruitment with no second event prior to data collection was relatively small, limiting the power of the study. A strength of our study is that in Australia treatment with disease modifying therapy (DMT) typically does not commence until a diagnosis of 
CDMS has been made. Our cohort is thus particularly valuable as the results on conversion to CDMS are not significantly confounded by treatment with DMT.

In conclusion, we provide evidence that a functional genetic variant in miR-146a, which has been linked in vitro with a change in miR-146a expression, predicts conversion to CDMS and relapse in MS. The combination of the risk genotype of miR-146a and higher levels of anti-EBNA-IgG result in a marked increase in risk of conversion to CDMS and relapse, suggesting that these two factors are component causes of a sufficient cause. These results are intriguing and suggest a potential novel risk locus, the precise mode of action, either independently or in association with EBV infection, that may influence disease progression following initial CNS demyelination needs to be substantiated by future functional studies. In particularly, these should include the relationship between rs2910164 in miR-146a and its target genes, and the influences on cytokine levels and potential signaling pathways.

\subsection{Acknowledgements}

The members of the AUSLONG investigators group are: Robyn M Lucas (National Centre for Epidemiology and Population Health, Canberra), Keith Dear (Duke Kunshan University, Kunshan, China), Anne-Louise Ponsonby and Terry Dwyer (Murdoch Childrens Research Institute, Melbourne, Australia), Ingrid van der Mei, Leigh Blizzard, Steve Simpson, Jr. and Bruce V Taylor (Menzies Institute for Medical Research, University of Tasmania, Hobart, Australia), Simon Broadley (School of Medicine, Griffith University, Gold Coast Campus, Australia), Trevor Kilpatrick (Centre for Neurosciences, Department of Anatomy and Neuroscience, University of 
Melbourne, Melbourne, Australia). David Williams and Jeanette Lechner-Scott

(University of Newcastle, Newcastle, Australia), Cameron Shaw and Caron

Chapman (Barwon Health, Geelong, Australia), Alan Coulthard (University of

Queensland, Brisbane, Australia) and Patricia Valery (QIMR Berghofer Medical

Research Institute, Brisbane, Australia).

\subsection{References:}

[1] D. Baek, J. Villen, C. Shin, F.D. Camargo, S.P. Gygi, D.P. Bartel, The impact of microRNAs on protein output, Nature 455(7209) (2008) 64-71.

[2] Y. Zhou, S. Simpson, Jr., A.F. Holloway, J. Charlesworth, I. van der Mei, B.V. Taylor, The potential role of epigenetic modifications in the heritability of multiple sclerosis, Mult Scler 20(2) (2014) 135-40.

[3] G. Liu, E. Abraham, MicroRNAs in immune response and macrophage polarization, Arterioscler Thromb Vasc Biol 33(2) (2013) 170-7.

[4] A. Junker, M. Krumbholz, S. Eisele, H. Mohan, F. Augstein, R. Bittner, H. Lassmann, H. Wekerle, R. Hohlfeld, E. Meinl, MicroRNA profiling of multiple sclerosis lesions identifies modulators of the regulatory protein CD47, Brain 132(Pt 12) (2009) 3342-52.

[5] A. Waschbisch, M. Atiya, R.A. Linker, S. Potapov, S. Schwab, T. Derfuss, Glatiramer acetate treatment normalizes deregulated microRNA expression in relapsing remitting multiple sclerosis, PloS one 6(9) (2011) e24604.

[6] S.E. Godshalk, S. Bhaduri-McIntosh, F.J. Slack, Epstein-Barr virus-mediated dysregulation of human microRNA expression, Cell cycle (Georgetown, Tex.) 7(22) (2008) 3595-600.

[7] K. Vrijens, V. Bollati, T.S. Nawrot, MicroRNAs as Potential Signatures of Environmental Exposure or Effect: A Systematic Review, Environ Health Perspect (2015).

[8] A. Ascherio, K.L. Munger, Environmental risk factors for multiple sclerosis. Part I: the role of infection, Ann Neurol 61(4) (2007) 288-99.

[9] A. Ascherio, K.L. Munger, Environmental risk factors for multiple sclerosis. Part II: Noninfectious factors, Ann Neurol 61(6) (2007) 504-13.

[10] J. Gong, Y. Tong, H.M. Zhang, K. Wang, T. Hu, G. Shan, J. Sun, A.Y. Guo, Genome-wide identification of SNPs in microRNA genes and the SNP effects on microRNA target binding and biogenesis, Human mutation 33(1) (2012) 254-63.

[11] R. Lucas, A.L. Ponsonby, K. Dear, B. Taylor, T. Dwyer, A. McMichael, P. Valery, A. van der Mei, D. Williams, M. Pender, T. Kilpatrick, C. Chapman, A. Coulthard, Associations between silicone skin cast score, cumulative sun exposure and other factors in the 
Ausimmune Study: a multicentre Australian study, Cancer Epidemiology Biomarker Prevention 18(11): 2887-94 (2009).

[12] R. Lucas, A.L. Ponsonby, A. McMichael, I. van der Mei, C. Chapman, A. Coulthard, K. Dear, T. Dwyer, T. Kilpatrick, M. Pender, B. Taylor, P. Valery, D. Williams, Observational analytic studies in multiple sclerosis: controlling bias through study design and conduct. The Australian Multicentre Study of Environment and Immune Function, Mult Scler 13(7) (2007) 827-39.

[13] K. Strautins, M. Tschochner, I. James, L. Choo, D.S. Dunn, M. Pedrini, A. Kermode, W. Carroll, D. Nolan, Combining HLA-DR risk alleles and anti-Epstein-Barr virus antibody profiles to stratify multiple sclerosis risk, Mult Scler 20(3) (2014) 286-94.

[14] C.H. Polman, S.C. Reingold, G. Edan, M. Filippi, H.P. Hartung, L. Kappos, F.D. Lublin, L.M. Metz, H.F. McFarland, P.W. O'Connor, M. Sandberg-Wollheim, A.J. Thompson, B.G. Weinshenker, J.S. Wolinsky, Diagnostic criteria for multiple sclerosis: 2005 revisions to the "McDonald Criteria", Ann Neurol 58(6) (2005) 840-6.

[15] W.I. McDonald, A. Compston, G. Edan, D. Goodkin, H.P. Hartung, F.D. Lublin, H.F. McFarland, D.W. Paty, C.H. Polman, S.C. Reingold, M. Sandberg-Wollheim, W. Sibley, A. Thompson, S. van den Noort, B.Y. Weinshenker, J.S. Wolinsky, Recommended diagnostic criteria for multiple sclerosis: guidelines from the International Panel on the diagnosis of multiple sclerosis, Ann Neurol 50(1) (2001) 121-7.

[16] J.F. Kurtzke, Rating neurologic impairment in multiple sclerosis: an expanded disability status scale (EDSS), Neurology 33(11) (1983) 1444-52.

[17] R.L. Prentice, B.J. Williams, A.V. Peterson, On the regression analysis of multivariate failure time data, Biometrika 68(2) (1981) 373-79.

[18] K. Jazdzewski, E.L. Murray, K. Franssila, B. Jarzab, D.R. Schoenberg, A. de la Chapelle, Common SNP in pre-miR-146a decreases mature miR expression and predisposes to papillary thyroid carcinoma, Proceedings of the National Academy of Sciences of the United States of America 105(20) (2008) 7269-74.

[19] Y. Li, C. Du, W. Wang, G. Ma, L. Cui, H. Zhou, H. Tao, L. Yao, B. Zhao, K. Li, Genetic association of MiR-146a with multiple sclerosis susceptibility in the Chinese population, Cell Physiol Biochem 35(1) (2015) 281-91.

[20] D. Khan, R. Dai, E. Karpuzoglu, S.A. Ahmed, Estrogen increases, whereas IL-27 and IFN-gamma decrease, splenocyte IL-17 production in WT mice, Eur J Immunol 40(9) (2010) 2549-56.

[21] R. Dai, R.A. Phillips, Y. Zhang, D. Khan, O. Crasta, S.A. Ahmed, Suppression of LPSinduced Interferon-gamma and nitric oxide in splenic lymphocytes by select estrogenregulated microRNAs: a novel mechanism of immune modulation, Blood 112(12) (2008) 4591-7.

[22] R. Park, W.J. Lee, J.D. Ji, Association between the three functional miR-146a singlenucleotide polymorphisms, rs2910164, rs57095329, and rs2431697, and autoimmune disease susceptibility: A meta-analysis, Autoimmunity (2016) 1-8.

[23] E.K. Chan, A. Ceribelli, M. Satoh, MicroRNA-146a in autoimmunity and innate immune responses, Annals of the rheumatic diseases 72 Suppl 2 (2013) ii90-5.

[24] D. Baumjohann, K.M. Ansel, MicroRNA-mediated regulation of T helper cell differentiation and plasticity, Nature reviews. Immunology 13(9) (2013) 666-78. [25] M.P. Pender, S.R. Burrows, Epstein-Barr virus and multiple sclerosis: potential opportunities for immunotherapy, Clin Transl Immunology 3(10) (2014) e27.

[26] G. Disanto, C. Hall, R. Lucas, A.L. Ponsonby, A.J. Berlanga-Taylor, G. Giovannoni, S.V. Ramagopalan, G. Ausimmune Investigator, Assessing interactions between HLA- 
DRB1*15 and infectious mononucleosis on the risk of multiple sclerosis, Mult Scler 19(10) (2013) 1355-8.

[27] E. Sundqvist, P. Sundstrom, M. Linden, A.K. Hedstrom, F. Aloisi, J. Hillert, I. Kockum, L. Alfredsson, T. Olsson, Lack of replication of interaction between EBNA1 IgG and smoking in risk for multiple sclerosis, Neurology 79(13) (2012) 1363-8.

[28] A.K. Hedstrom, I.L. Bomfim, L.F. Barcellos, F. Briggs, C. Schaefer, I. Kockum, T.

Olsson, L. Alfredsson, Interaction between passive smoking and two HLA genes with regard to multiple sclerosis risk, Int J Epidemiol 43(6) (2014) 1791-8. 


\section{Figure Title and Legend}

Figure 1: The predicted secondary structure of pre-miR-146a by rs2910164 polymorphism. The red represents the mature miR-146a duplex.

Figure 2 a) Kaplan-Meier survival plot for time to CDMS by category of rs2910164 genotype for those with a classical FDE.

Figure 2 b) Kaplan-Meier survival plot for time to relapse by category of rs2910164 genotype.

Figure 3: a) Hazard ratios for time to convert to CDMS by different combinations of baseline anti-EBNA-1 IgG titers (Low: $<=0.53$, High:>0.53) and miR-146a genotype $(\mathrm{GG}, \mathrm{GC}+\mathrm{CC})$. Test for interaction on the multiplicative scale, $\mathrm{HR}=1.48, \mathrm{p}=0.38$; test for interaction on the additive scale, RERI=2.39, $\mathrm{p}=0.00002$.

Figure $3 \mathrm{~b}$ ) Hazard ratios for time relapse by different combinations of baseline antiEBNA-1 IgG titers (Low: <=0.53, High:>0.53) and miR-146a genotype (GG, $\mathrm{GC}+\mathrm{CC}$ ). Test for interaction on the multiplicative scale, $\mathrm{HR}=1.16, p=0.82$; test for interaction on the additive scale, $R E R I=1.20, p=0.006$. 
Table1: Association between miR-146a SNP rs2910164 genotype and measures of clinical course.

\begin{tabular}{|c|c|c|c|}
\hline rs2910164 & $\mathrm{N}$ & CDMS (HR $(95 \% \mathrm{Cl}))$ & Relapse (HR (95\% CI)) \\
\hline GG & 93 & Ref & Ref \\
\hline \multirow[t]{2}{*}{$\mathrm{GC}+\mathrm{CC}$} & 58 & $1.52(0.97,2.42)$ & $2.10(1.43,3.08)$ \\
\hline & & $p=0.07$ & $P=0.0001$ \\
\hline \multicolumn{4}{|c|}{$\begin{array}{l}\text { Due to the small number of people carrying the CC genotype who converted to CDMS ( } N=1) \text {, we recoded the genotype as } \\
\text { GC+CC. Results were adjusted for age, sex and study site, and presented as HR ( } 95 \% \mathrm{Cl}) \text { for time to CDMS and relapses } \\
\text { The N refers to the number of rs2910164 genotype in the subgroup with a "classic FDE". } \\
\text { HR: Hazard ratio, Cl: Confidence interval, CDMS: clinical definite MS }\end{array}$} \\
\hline
\end{tabular}


Table2: Interaction between miR-146a SNP rs2910164 with baseline EBV antibody levels (anti-EBNA-1 and anti-EBNA-2) and according to history of having had infectious mononucleosis on time to conversion to CDMS and relapse.

\begin{tabular}{|c|c|c|c|c|c|c|c|c|}
\hline \multirow{3}{*}{$\begin{array}{l}\text { Clinical } \\
\text { Course }\end{array}$} & \multirow{3}{*}{$\begin{array}{c}\text { Environmental } \\
\text { exposure }(E)\end{array}$} & \multirow{3}{*}{ Genetic exposure (G) } & \multirow{2}{*}{$\begin{array}{l}\text { Effects of not } \\
\text { having factor } E \\
\text { and } G\end{array}$} & \multirow{2}{*}{$\begin{array}{l}\text { Effects of having } \\
\text { factor } E \text { in } \\
\text { absence of } \\
\text { factor } G\end{array}$} & \multirow{2}{*}{$\begin{array}{l}\text { Effects of having } \\
\text { factor } G \text { in } \\
\text { absence of factor } \\
\text { E }\end{array}$} & \multirow{2}{*}{$\begin{array}{l}\text { Effects of having } \\
\text { both factor } E \\
\text { and factor } G\end{array}$} & \multicolumn{2}{|c|}{ Interaction Parameters } \\
\hline & & & & & & & $\begin{array}{l}\text { Multiplicative } \\
\text { Interaction }\end{array}$ & $\begin{array}{c}\text { Additive } \\
\text { Interaction }\end{array}$ \\
\hline & & & $\mathrm{HR}_{\text {None }}(95 \% \mathrm{Cl})$ & $\mathrm{HR}_{\mathrm{E}}(95 \% \mathrm{Cl})$ & $\mathrm{HRG}_{\mathrm{G}}(95 \% \mathrm{Cl})$ & $\mathrm{HR}_{\mathrm{GE}}(95 \% \mathrm{Cl})$ & $\mathrm{HR}_{\text {multi }}(p)$ & $\operatorname{RERI}(p)$ \\
\hline \multirow{3}{*}{ CDMS } & High baseline EBNA1 & miR-146a (GC+CC) & $\begin{array}{c}\text { Ref } \\
N=44\end{array}$ & $\begin{array}{c}3.54(1.48-8.43) \\
N=42\end{array}$ & $\begin{array}{c}1.59(0.54-4.67) \\
N=28\end{array}$ & $\begin{array}{c}6.52(2.41-17.67) \\
N=24\end{array}$ & $1.48(0.38)$ & $2.39(0.00002)$ \\
\hline & High baseline EBNA2 & miR-146a $(G C+C C)$ & $\begin{array}{c}\text { Ref } \\
N=49\end{array}$ & $\begin{array}{c}1.62(0.76,3.46) \\
N=37\end{array}$ & $\begin{array}{c}1.09(0.40,2.94) \\
N=27\end{array}$ & $\begin{array}{c}2.49(1.09,5.66) \\
N=25\end{array}$ & $1.41(0.60)$ & $0.78(0.05)$ \\
\hline & $\begin{array}{c}\text { Infectious } \\
\text { mononucleosis }\end{array}$ & miR-146a (GC+CC) & $\begin{array}{c}\text { Ref } \\
N=69\end{array}$ & $\begin{array}{c}0.60(0.26,1.38) \\
N=22\end{array}$ & $\begin{array}{c}1.35(0.79,2.31) \\
N=39\end{array}$ & $\begin{array}{c}1.38(0.69,2.77) \\
N=19\end{array}$ & $1.69(0.34)$ & $0.43(0.21)$ \\
\hline \multirow{3}{*}{ Relapse } & High baseline EBNA1 & miR-146a $(\mathrm{GC}+\mathrm{CC})$ & $\begin{array}{c}\text { Ref } \\
\mathrm{N}=44\end{array}$ & $\begin{array}{c}1.23(0.69-2.17) \\
N=42\end{array}$ & $\begin{array}{c}1.75(0.90-3.40) \\
N=28\end{array}$ & $\begin{array}{c}3.18(1.69-5.97) \\
N=24\end{array}$ & $1.16(0.82)$ & $1.20(0.006)$ \\
\hline & High baseline EBNA2 & miR-146a $(G C+C C)$ & $\begin{array}{c}\text { Ref } \\
N=49\end{array}$ & $\begin{array}{c}0.87(0.49,1.54) \\
N=37\end{array}$ & $\begin{array}{c}1.22(0.68,2.19) \\
N=27\end{array}$ & $\begin{array}{c}2.65(1.56,4.52) \\
N=25\end{array}$ & $2.50(0.03)$ & $1.56(0.005)$ \\
\hline & $\begin{array}{c}\text { Infectious } \\
\text { mononucleosis }\end{array}$ & miR-146a $(\mathrm{GC}+\mathrm{CC})$ & $\begin{array}{c}\text { Ref } \\
N=69\end{array}$ & $\begin{array}{c}1.21(0.49,2.98) \\
N=22\end{array}$ & $\begin{array}{c}1.72(1.15,2.58) \\
N=39\end{array}$ & $\begin{array}{c}3.40(2.24,5.17) \\
N=19\end{array}$ & $2.53(0.09)$ & $1.47(0.0006)$ \\
\hline
\end{tabular}

High baseline EBNA1 status ( $>0.53$ vs <=0.53); High baseline EBNA2 status( $>0.70$ vs $<=0.70$ ); Infectious mononucleosis (yes vs no); $m i R 146 a$ (GC $+C C$ vs $G G)$

CDMS: Clinical diagnosis of multiple sclerosis; HR: hazard ratio; Cl: confidence interval; RERI: relative excess risk due to interaction on additive scale; $p: p$ value

The $\mathrm{N}$ refers to the number of rs2910164 genotype in the subgroup with a "classic FDE". 